\title{
rTMS for tinnitus
}

\section{Robert L. Folmer*}

National Center for Rehabilitative Auditory Research, Portland VA Medical Center, Portland, OR, USA

*Correspondence: folmerr@ohsu.edu

\section{A commentary on}

Can temporal repetitive transcranial magnetic stimulation be enhanced by targeting affective components of tinnitus with frontal rTMS? A randomized controlled pilot trial

by Kreuzer, P. M., Landgrebe, M., Schecklmann, M., Poeppl, T. B., Vielsmeier, V., Hajak, G., Kleinjung, T., and Langguth, B. (2011). Front. Syst. Neurosci. 5:88. doi: 10.3389/ fnsys.2011.00088

I applaud the authors of the article "Can temporal repetitive transcranial magnetic stimulation be enhanced by targeting affective components of tinnitus with frontal rTMS? a randomized controlled pilot trial" because many of them are pioneers in the development of protocols that use rTMS to treat chronic tinnitus. Investigators (including myself) around the world conducting similar studies of rTMS for tinnitus often follow the procedural examples of these researcher-clinicians in Germany. In this recent publication, Kreuzer et al. (2011) compared the effects of 2000 daily pulses of rTMS delivered to patients' left temporal region with a protocol that began with 1000 pulses of right frontal rTMS followed by 1000 pulses of left temporal rTMS. In both protocols, patients exhibited improvement in measures of tinnitus severity. However, there was no significant difference in treat- ment outcomes between the left temporal and the combined (right frontal + left temporal) rTMS protocols. I would like to add a few comments about this study:

(1) No placebo condition was included. Because the placebo effect can be significant for many tinnitus patients (Dobie, 1999), a placebo condition should be used in most clinical trials involving this population.

(2) The fact that patients' scores for the Tinnitus Questionnaire, THI, and BDI all declined from the time of screening to the time just before the first rTMS session (baseline) illustrates, I believe, the placebo effect (or, as the authors describe it, "an anticipation effect"). This decline in scores from screening to baseline is similar in magnitude to the decline in scores that occurred during rTMS treatment.

(3) The authors state that they used a left temporal coil placement for rTMS (regardless of the location of patients' tinnitus perception) in order to compare results with previous studies that used the same coil placement. However, because Frank et al. (2010) reported that left temporal rTMS was not effective for patients with rightside tinnitus, it is time for researchers to re-evaluate rTMS coil placement in experimental designs.
All of us conducting rTMS studies for tinnitus realize that many procedural questions need to be addressed before this treatment option can reach its full clinical potential. We thank our colleagues in Germany for continuing to conduct studies that increase our understanding and contribute to improvements in this area of research.

\section{REFERENCES}

Dobie, R.A. (1999). A review of randomized clinical trials in tinnitus. Laryngoscope 109, 1202-1211.

Frank, G., Kleinjung, T., Landgrebe, M., Vielsmeier, V., Steffenhagen, C., Burger, J., Frank, E., Vollberg, G., Hajak, G., and Langguth, B. (2010). Left temporal low-frequency rTMS for the treatment of tinnitus: clinical predictors of treatment outcome-a retrospective study. Eur. J. Neurol. 17, 951-956.

Kreuzer, P. M., Landgrebe, M., Schecklmann, M., Poeppl, T. B., Vielsmeier, V., Hajak, G., Kleinjung, T., and Langguth, B. (2011). Can temporal repetitive transcranial magnetic stimulation be enhanced by targeting affective components of tinnitus with frontal rTMS? A randomized controlled pilot trial. Front. Syst. Neurosci. 5:88. doi: 10.3389/ fnsys.2011.00088

Received: 20 October 2011; accepted: 02 January 2012; published online: 18 January 2012.

Citation: Folmer RL (2012) rTMS for tinnitus. Front. Hum. Neurosci. 6:1. doi: 10.3389/fnhum.2012.00001

Copyright $\odot 2012$ Folmer. This is an open-access article distributed under the terms of the Creative Commons Attribution Non Commercial License, which permits non-commercial use, distribution, and reproduction in other forums, provided the original authors and source are credited. 